\title{
PI3Ka/mTOR Inhibitor PKI-179
}

National Cancer Institute

\section{Source}

National Cancer Institute. PI3Ka/mT OR Inhibitor PKI-179. NCI Thesaurus. Code C90291.

A second generation, small-molecule mimetic of ATP that targ ets the mammalian target of rapamycin (mTOR) with potential antineoplastic activity. PKI-179 selectively inhibits mTOR and phosphoinositide-3-kinase (PI3K) alpha. By inhibiting the PI3K/mT OR signaling pathway, this agent may inhibit tumor cell proliferation and survival. 